DOI: /((please add manuscript number $))$

Type: Article

\title{
Nano-porous substrate-infiltrated hydrogels: a bio-inspired regenerable surface for high load bearing and tunable friction
}

Shuanhong Ma, M. Scaraggi, Daoai Wang, Xiaolong Wang, Yongmin Liang, Weimin Liu, D. Dini* and Feng Zhou*

Dr. S. Ma, Prof. D. Wang, Prof. X. Wang, Prof. Y. Liang, Prof. W. Liu, Prof. F. Zhou

State Key Laboratory of Solid Lubrication, Lanzhou Institute of Chemical Physics, Chinese

Academy of Sciences, Lanzhou, 730000, China

E-mail: zhouf@licp.cas.cn

Dr. S. Ma,

China University of the Chinese Academy of Sciences, Beijing, 100049, China

Dr. M. Scaraggi,

DII, Universitá del Salento, 73100 Monteroni-Lecce, Italy, EU

Dr. D. Dini

Imperial College London, United Kingdom, EU

E-mail:d.dini@imperial.ac.uk

Abstract: Nature has successfully combined soft matter and hydration lubrication to achieve ultra-low friction even at relatively high contact pressure (e.g. articular cartilage). Inspired by this, scientists have used hydrogels to mimic natural aqueous lubricating systems. However, hydrogels usually cannot bear high load because of solvation in water environments and are, therefore, not adopted in real applications. In this work, we developed a novel composite surface of ordered hydrogel nanofiber arrays confined in anodic aluminum oxide (AAO) nanoporous template based on a soft/hard combination strategy. The synergy between the soft hydrogel fibers, which provide excellent aqueous lubrication, and the hard phase AAO, which gives high load bearing capacity, is shown to be capable of attaining very low coefficient of friction (< 0.01) under heavy load (with mean contact pressures in the $2 \mathrm{MPa}$ range). Interestingly, the composite synthetic material was very stable and could not be peeled off during sliding and exhibited the desirable regenerative (self-healing) properties, which can assure long term resistance to wear. Moreover, the crosslinked polymethylacrylic acid (PMAA) hydrogels were shown to be able to promptly switch between high friction $(>0.3)$ and superlubrication $\left(\sim 10^{-3}\right)$ when their state was changed from contracted to swollen by means of acidbasic actuation. The mechanisms governing ultra-low and tunable friction are theoretically explained via an in-depth study of the chemo-mechanical interactions responsible for the behavior of these substrate-infiltrated hydrogels. These findings open a promising route for the design of ultra-slippery and smart surface/interface materials.

Keywords: Nano-porous, Gel fibers array, Tunable friction/lubrication, Regenerable surface, Bio-mimicking, Hydrogel lubrication 


\section{Introduction}

The understanding of friction and contact mechanisms occurring in biological systems has inspired much research activity in the last few decades. Just to cite a few examples, wax crystals on the top of micro-bumps are now known to produce very effective self-cleaning property in Lotus leaf, ${ }^{[1-4]}$ whilst the multiscale contact splitting nature of the Gecko foot, originating from the superposition of micro-scale spatulae covered by anti-self-sticking setae, allows the lizard to reversibly adhere on almost any rough surface. ${ }^{[5-7]}$ Nature provides a plethora of contact surfaces optimized over many years, ${ }^{[8]}$ mainly linked to the evolution required to meet very different survival strategies. ${ }^{[9]}$

However, in biological systems one cannot treat interfacial mechanics solely as a matter of friction, adhesion and wear. A powerful example of how biology and contact mechanics are intimately linked is cell signalling, a mesoscale phenomenon which is fundamentally governed by cell wall-stresses and rules the cell-cell as well as the cell-extracellular matrix (ECM) interactions in many soft tissues. ${ }^{[10]}$ Surface interactions and stresses can strongly affect the cell fate; the presence of a surface pattern (e.g. rough surfaces ${ }^{[11,12]}$ or the introduction of stiff superficial layers ${ }^{[13]}$ ) can rule the cell growth/multiplication/apoptosis in a remarkably controllable fashion. In particular, healthy cartilage is a typical example of tissue whose environment provides the perfect match between ultra-low friction and stress-driven cell differentiation. ${ }^{[14]}$ In recent years, the hydration-lubricated soft contact characteristics of such tissue has attracted significant research interest, ${ }^{[15-20]}$ mainly focussed on mimicking its ultraslippery characteristics. Ultra-low friction is usually difficult to achieve in both oil (with very limited exceptions ${ }^{[21]}$ ) and water lubricated systems (due to its low viscosity value). Nevertheless, nature has favoured the development of structures that utilize aqueous lubrication to achieve very low friction whilst withstanding relatively high loads (e.g. $\sim \mathrm{MPa}{ }^{[22-23]}$ for 
articular cartilage) thanks to the water entrapment mechanisms (by wetting dynamics or because of bulk porosity) at the interface.

In the past few years, despite the vast research conducted in this area, the quest for ultralow friction artificial contact pairs has produced results mainly limited to ideal laboratory conditions. ${ }^{[24-25]}$ Very challenging tasks still remain for biologists, chemists and materials scientists in the strenuous search for the optimal ultra-low friction solution. ${ }^{[24,26,27]}$ Hydrogels are potential candidates for artificial cartilage materials, as they are characterized by intrinsically-low friction due to their strong hydration capability. ${ }^{[28-31]}$ However, excessive hydration, often occurring under physiological environment, usually drastically reduces the hydrogels bearing capability. ${ }^{[28]}$ Moreover, once damaged, the interfacial lubricating layer is disrupted and the system is usually incapable of self-healing. The capability of a tissue construct to self-heal is a highly desirable characteristic of any newly developed lubricating pair, ${ }^{[32-34]}$ which must be also capable of significantly lowering friction at high contact pressures.

A possible solution path is biomimicking. As an example, the epidermal mucus of fish skin could produce ultra-low friction owing to the excellent hydrophilicity of some of its aqueous proteins constituents and to the fast mucus ejection capability; ${ }^{[35-36]}$ in the particular case of eel skin we found a friction coefficient $\mu<0.01$ (see supporting information, S1) when it is still attached on muscle tissue (while a separate piece of epidermis showed an increased friction coefficient.). Inspired by the result, here we attempted to mimic the eel composite interface by engineering nanopores as the reservoir for hydrogels that can be released once the surface layer has been removed, therefore keeping a hydrated, regenerable lubrication state. In particular, the composite surface is made of an ordered hydrogel nanofiber array confined in an anodic aluminum oxide (AAO) template (the reservoir) with ejection capabilities. In this soft/hard combination strategy, the soft hydrogel layer provides excellent aqueous lubrication whereas the AAO hard phase (the nano-pillar porous reservoir) can bear high load. 


\section{Result and Discussion}
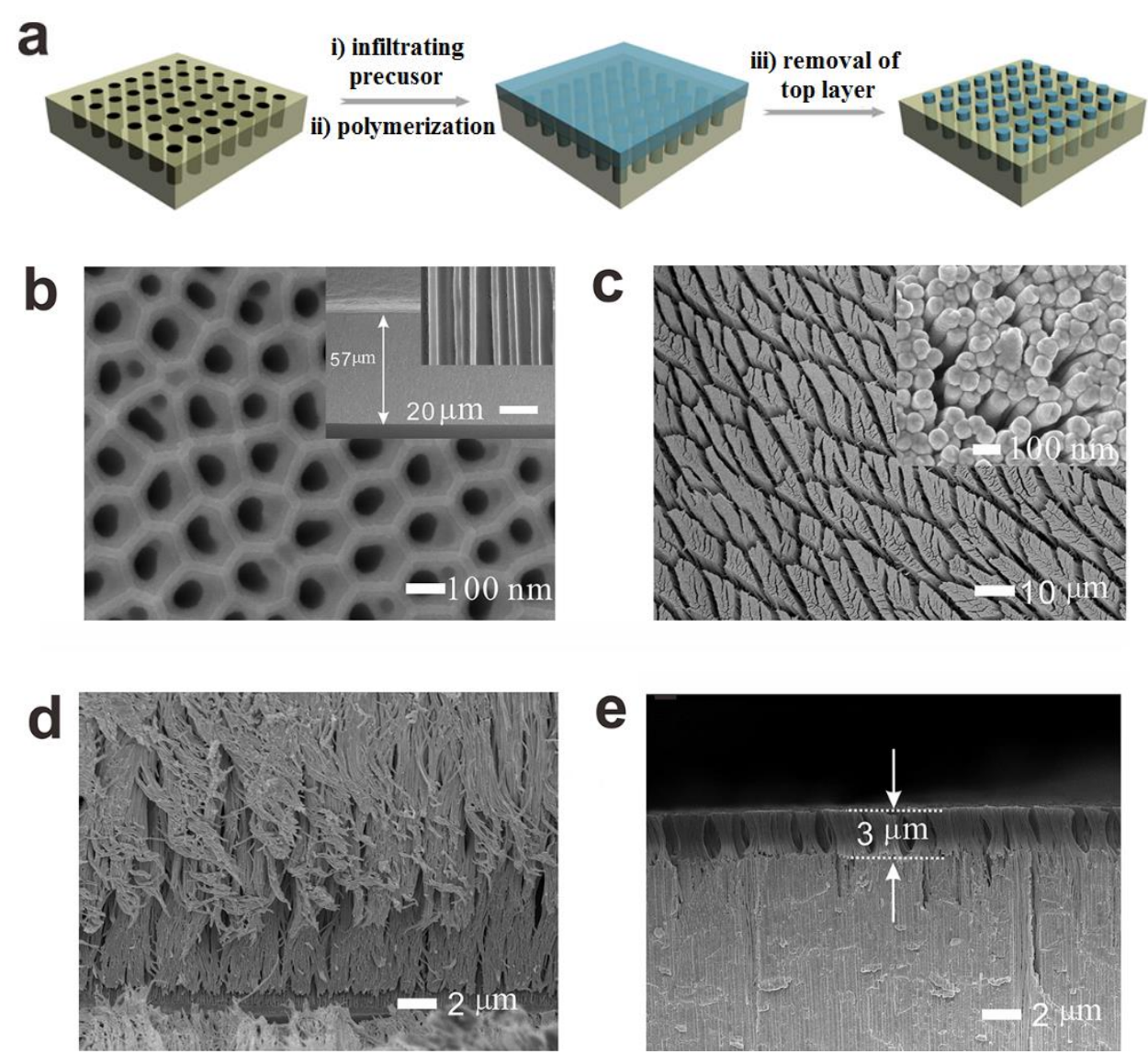

Figure 1: (a) Schematic of the preparation process of hydrogel nanofibers embedded into AAO.

FESEM images of AAO template (b), top view of the as-prepared PAA hydrogel nanofibers array (c), bottom part of confined PAA hydrogel nanofibers (d), and cross-sectional PAA hydrogel nanofibers array including protrusions out of nanopores after removal of surface bulk gel layer (e).

Preparation of hydrogel fiber arrays is depicted in Figure 1a. A mixture of monomer acrylic acid (AA), crosslinker N,N'-methylene bis(acrylamide) (BIS), initiator potassium persulfate (APS) and solvent was poured onto AAO membrane (see $\mathrm{S} 2$ for preparation process). Then free radical polymerization was initiated and followed by removing the bulky gel layer and the ordered gel fiber arrays underneath were formed. The top of the AAO membrane has the funnel structure with disordered hexagonal outer edge of about $100 \mathrm{~nm}$ and the inner pore diameter of 65-80 nm, while the cross-section observation indicates these nanopores are well 
aligned (Figure 1b). After peel-off of the top gel layer, well-defined gel fiber arrays were formed (Figure 1c). In detail, the top gel layer was more liable to lose water and shrink; this induced a weak break-up point on the connection area, which made them easy to be peeled off from the underlying substrate and gel (Figure S3). The thickness and diameter of nanopores can be tuned by varying anodization time and should determine the amount of storing hydrogels. Unless specified, the thickness of AAO layer and of gel fibers used in the paper is about $57 \mu \mathrm{m}$. These gel fibers were separated from each other even on top and had an average diameter size of $\sim 100$ $\mathrm{nm}$, a slightly larger than the diameter of nanopores. The areal density is $5.4 \times 10^{7} / \mathrm{mm}^{2}$ (the gel occupancy fraction is calculated to be $43 \%$ ). For comparison, a surface with the low areal fiber density $\left(4.0 \times 10^{7} / \mathrm{mm}^{2}\right.$, the gel occupancy fraction $\left.\left.17 \%\right)\right)$ was also prepared. Cross-section analysis indicates that the whole nanopores were filled in with hydrogels (see the bottom part of fibers in Figure 1d) and the overall length of gel fibers arrays were about $57 \mu \mathrm{m}$ while those outside nanopores had uniform length of only $3 \mu \mathrm{m}$ (Figure 1e). The contrast fiber length outside and inside nanopores ( $3 \mu \mathrm{m}$ vs $57 \mu \mathrm{m}$ ) is the basis of self-regeneration and long term functioning since it is very likely that the inner hydrogel fibers will continuously swell out of nanopores.

Figure 2 shows typical contact images obtained for the sliding pairs and the corresponding friction curves. Friction tests were performed under ball-on-disc contact, by adopting a soft ball (Young's modulus 2.0 MPa) of polydimethysiloxane (PDMS) elastomer with diameter of $6 \mathrm{~mm}$ sliding onto the composite interface. In order to explore the robustness of the interface to varying contact conditions, friction measurements were performed for different hydrogel thicknesses, loads, sliding velocities and composition (crosslinking degree), under constant weak basic medium $(\mathrm{pH}=10)$ as wetting liquid (unless differently specified). The effect of hydrogel thickness on the bearing capacity and friction was investigated for both the hydrogel $\mu \mathrm{m}$-thin slab (Figure $2 \mathrm{a}$ ) and bulk (thicker) gel samples (Figure $2 \mathrm{c}$ ). Figures $2 \mathrm{~b}$ and $2 \mathrm{~d}$ show the corresponding time evolution of friction coefficient at, respectively, $40 \mathrm{~N}$ and 
$3 \mathrm{~N}$ normal loads. Except for a short running-in period at the beginning, the sliding on hydrogel fibers soon turned to stable friction with extremely low value $(\mu \approx 0.007)$, much lower than the
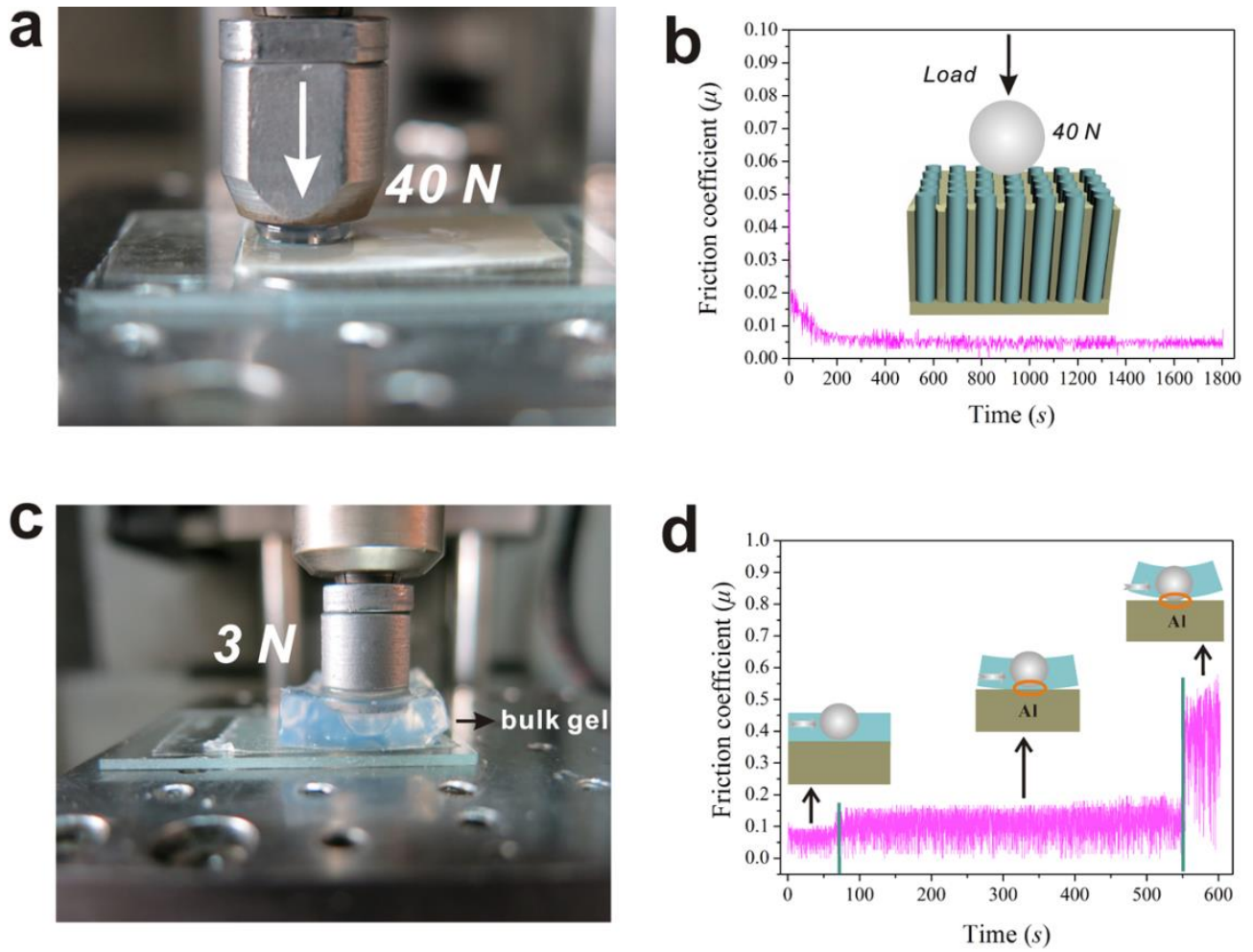

Figure 2: (a)-(b) PAA nanohydrogel array at a load of $40 \mathrm{~N}$ and sliding velocity of $0.01 \mathrm{~m} / \mathrm{s}$ in weak basic media ( $\mathrm{pH}=10)$, and typical corresponding friction curve. (c)-(d) Bulk hydrogel on aluminum at a load of $3 \mathrm{~N}$ and sliding velocity of $0.01 \mathrm{~m} / \mathrm{s}$, and typical corresponding friction curve.

sliding friction against blank AAO, $\mu>0.5$, where the PDMS registered severe wear after few sliding cycles) for the $\mu$ m-thin slab. For the bulky gel, inversely, we found that even at much lower applied load of only $3 \mathrm{~N}$, the PDMS ball penetrated into the gel (Figure 2c). A thin gel film exhibited much higher friction than the confined hydrogel, showing a value of $\approx 0.1$ due to the ploughing action of the sliding ball, to finally reach a hydrogel worn stage, which leads to the rapid rise of friction coefficient due to the PDMS/AAO contact (insets, Figure 2d). 
In general, the friction of hydrogels system could be typically attributed to three terms: (1) viscoelastic dissipation from the interfacial interaction; (2) elastic deformation from contact plough under high load; (3) viscous dissipation from the lubricant itself. As discussed in the section describing the theoretical model, friction in confined hydrogels is based on different mechanisms depending on the (hydrogel) coating size and lubrication regime. Whilst for bulk interactions a wear-driven plough action cannot be avoided, for a coating size of $\sim 1 \mu \mathrm{m}$ ultralow friction can be obtained even at high bearing loads (relatively to classical soft interactions load values). In particular, at relatively high sliding speeds, an effective aqueous interfacial separation is generated as a consequence of the confined imbibition/ejection of solution through the hydrogel, a process which is driven by the sliding action. As demonstrated in the following, dissipation here is not originated from the water-film shearing action, but the effective visceolastic nature of such imbibition/ejection process, which has an irreversible nature. Furthermore, friction can switch from ultra-low to high values under low sliding speed by regulating the local hydration process, which in turn affects the Schallamach-like friction process between sliding polymers. Such mechanisms are complemented by the fact that the confinement from porous AAO substrate greatly strengthens the interfacial bonding force of gel to substrate, which would prevent the gel from being peeled off under high load shearing. Further, the dense gel nanofibers layer generates high counter-ion osmotic pressure which finally separates two sliding surfaces. All of these lead to ultra-low friction of the composite interface in sliding. 

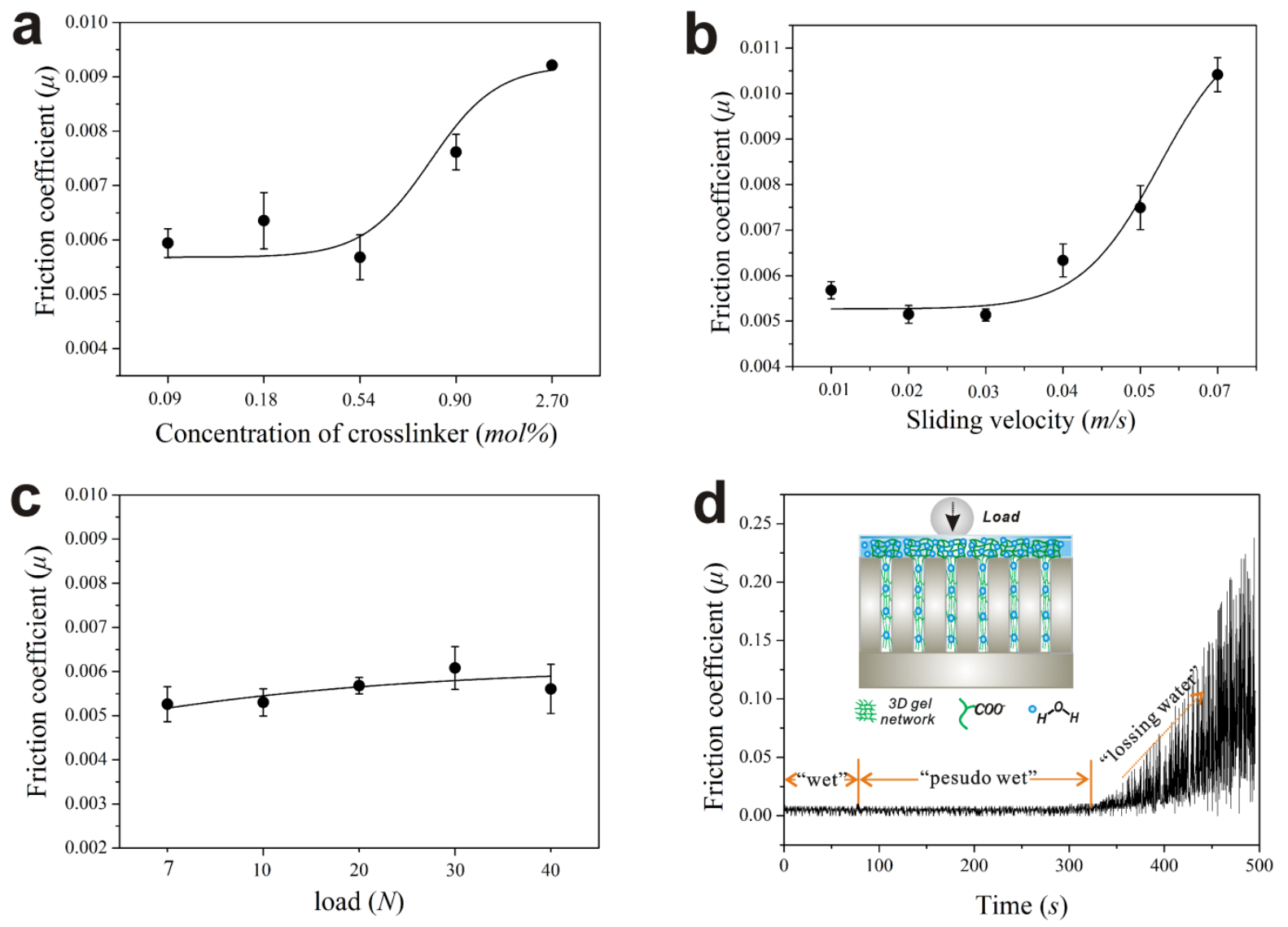

Figure 3: Friction coefficient of nanoconfined PAA nanohydrogel array as a function of (a) crosslinking degrees (at $v_{0}=0.01 \mathrm{~m} / \mathrm{s}$ and $F_{0}=20 \mathrm{~N}$ ); (b) for different sliding velocities (at $F_{0}=20$ $\mathrm{N})$ and (c) as a function of applied load (at $v_{o}=0.01 \mathrm{~m} / \mathrm{s}$ ) at crosslinking degrees of $0.54 \mathrm{~mol} \%$. (d) Friction curve for single droplet lubrication, showing the friction evolution during the water evaporation process. The inset shows the distribution of water molecules at interface.

At low crosslinking degree $(<0.5 \mathrm{~mol} \%$ : crosslinker fraction), ultra-low friction, $\mu \approx$ 0.005, was observed (Figure 3a). A further increase of the crosslinking degree resulted in a gradual increase of friction (as expected ${ }^{[29]}$ ), due to the increase of the hydrogel elastic modulus and to the consequent reduction of the degree of swelling. Constant or slightly decreasing friction was recorded for increasing speeds at low frequencies; the value of the coefficient of friction increased from 0.005 to 0.01 while the sliding speed changed from 0.03 to $0.07 \mathrm{~m} / \mathrm{s}$ (Figure 3b). This apparently hydrodynamic Stribeck regime is not compatible with the dissipation occurring during the shearing process of the interfacial thin water layer. Figure $3 \mathrm{c}$ 
shows the friction at different applied loads, for a sliding speed of $0.01 \mathrm{~m} / \mathrm{s}$. It is clear that the friction coefficient on the confined hydrogels remained very low and did not change much when the normal load changed from $7 \mathrm{~N}$ to $40 \mathrm{~N}$. It was found that the mean contact pressure can reach up to $1.8 \mathrm{MPa}$, much higher than that conventional hydrogels can withstand, which is normally at $\mathrm{kPa}$ level that is far from the bearing physiological pressure experienced by human cartilaginous joints (in the 5-10 MPa range ${ }^{[28]}$ ). Further, friction coefficients were recorded during a process of water evaporation/dehydration under high load (40 N). As shown in Figure $3 \mathrm{~d}$, the friction coefficient was very low and stable even after the presence of water was not observed around the contact. The low friction pseudo-wet process could even last around 350s, longer than water evaporation duration. The low friction is probably due to the migration of water stored in the AAO pores to the top surface (inset). This super-lubricity state has never been observed in bulk hydrogels, and it is related to the beneficial water ejection effects of nanopores, which act as a lubricant reservoir during boundary lubrication, caused by the sliding motion.

a

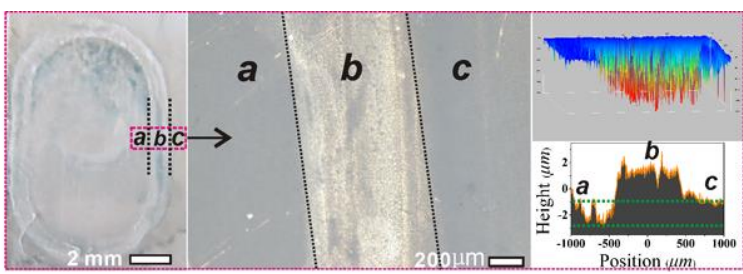

b

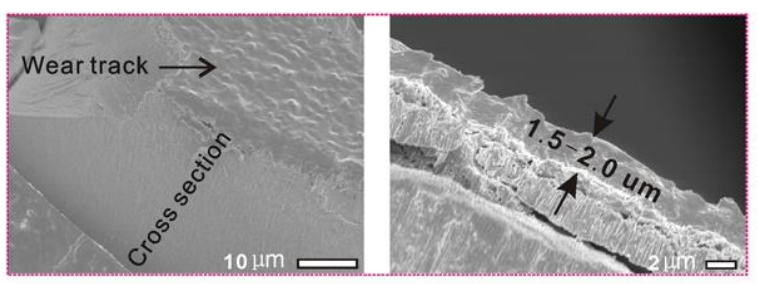

d

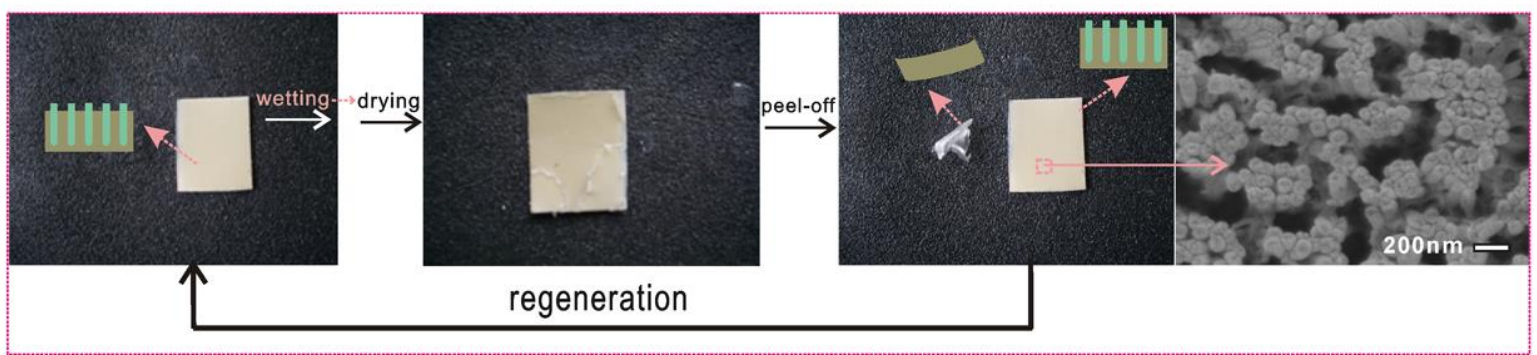

Figure 4: (a) Morphology of wear region- $a$, boundary region- $b$ and non-wear region- $c$, leftoptical image, middle-optical microscope image and right-corresponding profile image in 
confocal laser scanning microscope (CLSM). (b) Morphology of the test tracks of nanohydrogel array after sliding for 10800 cycles in continuous various loads at sliding velocities of $0.01 \mathrm{~m} / \mathrm{s}$, left-surface and right-cross section. (c) Morphology of the nanohydrogel array, top-fresh array surface assisted by nitrogen beam and bottom-after soaking in water for 45 days. (d) Regeneration process of gel fibers arrays, from left to right, wetted by immersing into weak basic media, peel off by tweezers in dehydration process, and the new regenerated surface with corresponding morphology.

Figure 4a shows the abrasion mark after friction test. After the application of high normal and tangential loads, the edge (marked as b) of the test region (marked as a) uplifts obviously as seen from optical and microscopic characterization, in comparison with the noncontact region (marked as c). The three-dimensional imaging and profile calculation from confocal laser scanning microscope show the gel layer thickness decreases obviously after friction test. Detailed characterization of wear track is shown in Figure 4b. It is seen that after testing, the ordered gel array structure disappears concurrent with the appearance of the denser gel film (left, surface) with thickness of 1.5-2.0 um (right, cross-section). For analysis, after sufficient swelling, the gel fibers become highly hydrated and soften. Once the sliding begins, the high normal pressure would prompt to the gradual loss of the cross-link of the polymer gel network under heavy shear, and this wear process finally leads to the merge gel fibers into polymer gel film. As a result, the thickness of the gel layer ( $3 \mathrm{um}$ ) outside of the AAO is compressed to 1.5-2 um. In this case, the denser gel film formed within eh structure is characterised by charge density and dense hydration film responsible for high counter-ion osmotic pressure, ${ }^{[28]}$ and then the composite interface acts as a good lubricator. Importantly, this dense gel layer is able to steadily withstand a continuous load under $1 \mathrm{~Hz}$ reciprocating frequency for 10800 cycles (end of experiment), with low wear volume. This means that the formed dense gel film could be tightly confined into the pore, otherwise it would be easily sheared off from the interface. Further, it was found that the gel film could show good 
lubrication properties both in low concentration $(10 \mathrm{mM})$ and moderate concentration (1000 $\mathrm{mM}) \mathrm{NaCl}$ solution, while the friction coefficient increased slightly in extreme high concentration $(5000 \mathrm{mM})$ due to charging screening effect resulting from polymer collapse (Figure S4).

Further, this strong confining effect could be indirectly proved from a swelling test by immersing the material into pure water. As shown in Figure 4c, after immersing into water for 45 days, no gel fibers are observed to escape from the interface and the thickness variation could be negligible. Interestingly, after immersing the film into basic media for swelling and subsequent dehydration, the softened gel layer (Figure S5) could be peeled off from the interface and a fresh gel fibers layer is reproduced, demonstrating very good regenerative properties (Figure 4d).

Based on the results above, one of the key to achieve low friction in our system is the formation of dense viscous layer of polymer gel after destroying the ordered gel array structure.

For comparison, low-density PAA gel fibers array was also tested. As shown in Figure S5a-b, the low density gel fibers above the pores aggregate because of capillary force in deswelling process and also larger separation distance, which corresponds to large free-volume (Figure S5a-b). Further, due to the strong capillary force, it was found that part of the gel fibers were peeled off from the substrate or separate from that confined in the pores (Figure S6c). As a consequence, the composite array was not able to provide good lubrication, resulting in higher friction coefficient of $\sim 0.05$ (Figure S7). Reducing the distribution density results in shearing off the gel fibers from the substrate, and the subsequent formation of a discontinuous gel film from the broken gel particles (Figure S8). The preliminary experiment indicates that the gel fibers density plays a key role in lubrication of this composite system and systematic study of this effect will be explored in the future.

More important, this novel soft/hard composite interface provides huge potential for obtaining large-scale friction switching in situ. In fact, hydrogel can nowadays be designed to 
be sensitive to environment stimuli such as $\mathrm{pH}$, light, temperature, magnetic field. ${ }^{[37,38]}$ However, whilst much research interest has been focussed on the chemical and physical response of bulk hydrogel, little consideration has been provided on the capability of in situ switching between high friction and super-lubrication; such important feature has the huge potential to enable the development of usable smart-intelligent devices (e.g. motors) in chemical actuators. ${ }^{[39]}$ Figure 5a shows the evolution of friction coefficient with the rising $\mathrm{pH}$ value, the composite interface presents high friction in media below pKa of PAA while shows low friction in media below the pKa of PAA. The frictional signals are unstable in weak acid media (Figure S9). Then in situ friction switching is performed in the composite interface, by alternatively wetting with the weak acid $\left(\mathrm{pH}=3\right.$, below the $p K_{a}$ of PAA) media and weak basic media $(\mathrm{pH}=10$, above the $p K_{a}$ of PAA). It is observed that the switching is very fast (Figure 5b). Further, in situ monitoring the morphology evolution of gel fibers in different media by atomic force microscopy (AFM), shows that fibers are in highly hydrated state with smooth topography in $\mathrm{pH}=10$ media (Figure $5 \mathrm{c}$ ) while are agglomerated together with collapse state in $\mathrm{pH}=3$ media (Figure 5d). 

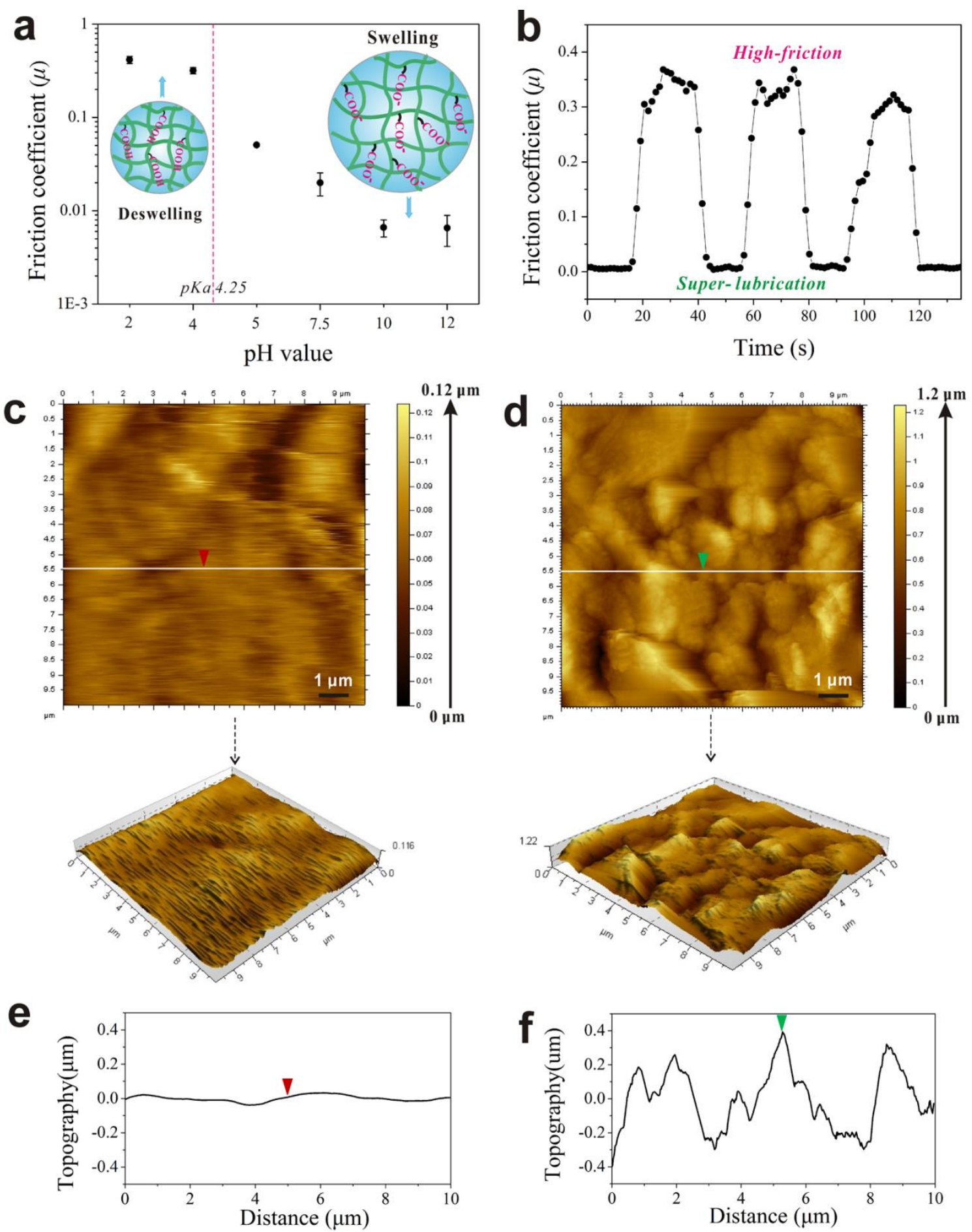

Figure 5: (a) Plots showing the response of friction coefficient in media with different $\mathrm{pH}$ values and corresponding deswelling and swelling mechanism of polymer chains. (b) Plots showing the sensitive switching of friction signals by alternatively wetted with acid and basic media. (c) AFM topography of the gel fibers in weak basic media $(\mathrm{pH}=10)$, and (d) weak acid media ( $\mathrm{pH}=3$ ). (e), (f) Corresponding tracking curve of cross section roughness profiles. (Load of $3 \mathrm{~N}$; sliding velocity $v_{0}=0.01 \mathrm{~m} / \mathrm{s}$ ) 


\section{Theoretical model}

This novel soft/hard composite interface presents unique lubrication features as demonstrated through the systematic experimental investigation presented above. However, the specific lubrication mechanisms responsible for such behaviour have not yet been unveiled. Here, a new theoretical model is developed, which is used to reveal the underlying phenomena governing lubrication and friction in the composite interface, by adopting scaling arguments (Figure 6). In typical case, a soft material (PDMS in our case) ball is pressed and placed in wet sliding contact against a $\mu \mathrm{m}$-thin hydrogel slab, the latter bonded on the rigid AAO substrate (Figure 6a). Moreover, the contact pair is immersed in the water solution, which is assumed to behave as an isothermal bath of both ions and solvent molecules. For this system, in the Supplemental Section 4 we derive an approximate theory of hydrogel lubrication, for both the static and steady-sliding interactions (both boundary and hydrodynamic regime). The theory allows establishing the lubrication regime as well as the friction value given, in particular, the initial hydrogel thickness and the solution $\mathrm{pH}$.

Let us focus first on the contact occurring under hydrodynamic conditions. By adopting the scaling arguments presented in Supplemental Section 4 one can show that a hydrodynamic lubrication regime (see e.g. Figure $3 b$ ) exists only for a hydrogel thickness $t<t_{\text {lim }}$. For our system we find $t_{\text {lim }} \approx 1$ to $10 \mu \mathrm{m}$, in agreement with the experimental findings. In such conditions, typical values of hydrodynamic friction $\mu_{\mathrm{EHL}}$ caused by the fluid shearing during lift can be estimated to be of order $10^{-4}$ (due to the low water viscosity), in neat disagreement with the friction reported in the hydrodynamic stage of Figure $3 b$ for the basic bath. However, soft contacts are characterized by several contributions to friction. ${ }^{[40]}$ For our system, a further source of dissipation is generated during the (sliding) contact-induced gel deswelling (the gel 
moves from a swollen state during no contact, to a less hydrated state under the contact, see Figure 6b).

This deswelling phenomenon is ruled by the relaxation dynamics occurring in the hydrogel, producing a hydrogel sliding friction $\mu_{\text {gel }} \propto \tan \delta$, where $\tan \delta$ is the deswelling loss tangent. For our system, in Figure $6 \mathrm{c}$ we report both $\mu_{\text {gel }}$ (black line) and $\mu_{\mathrm{EHL}}$ (red) as a function of the sliding velocity, together with the experimental findings of Figure $3 \mathrm{~b}$. The very good match between experimental and theoretical results confirms the picture that the hydrogel lubrication and friction mechanisms, during a hydrodynamic sliding contact, can be strongly different from classical soft contact mechanisms, since the contact-induced deswelling process introduces an ultra-low friction bearing capability which would not be possible with low viscosity soft contact lubrication.

For decreasing sliding velocities the contact moves toward the boundary regime, where the interaction is dominated by multiple asperity-asperity (random) contacts. The surface roughness has a $\mathrm{pH}$ dependence as shown in Figure $5 \mathrm{c}, 5 \mathrm{~d}$, resulting in a true contact shear stress $\tau \approx 2.3 \mathrm{MPa}$ for the acid solution (where $\mu=0.33$ ), and $\tau \approx 10 \mathrm{kPa}$ for the basic solution $(\mu=0.0077)$. Whilst for the acid-pH case the calculated true shear stress is comparable to those values typically encountered in soft (polymers) interactions, the two-orders of magnitude reduction characterizing the basic-pH bath has to be related to a hydration process. In our system, the PDMS surface may be partially oxidized as a consequence of exposure, hence its surface potential will be close to values reported in Figure 6d (PDMS curves). In Figure 6d we also report a hydrogel surface potential (PCDME) which we use to approximately describe our AA-hydrogel $\zeta$-potential. The $\mathrm{pH}$-regulated electrostatic interaction (proportional to the electrostatic repulsion $p_{\mathrm{r}}$ ) between gel and PDMS surface is proportional to the product of their surface potential $\zeta_{\text {hydro }} \zeta_{\text {PDMS }} \cdot{ }^{[41]}$ The latter is shown in Figure $6 \mathrm{~d}$ as a function of the solution $\mathrm{pH}$. We first observe that the $\mathrm{pH}$-driven electrostatic interaction is almost unaffected by the 
accurate description of the PDMS surface state. Moreover, a repulsive state occurs beyond the isoelectric point $\mathrm{pH} \approx 9$, which should correspond to a double layer formation, and hence to a hydration lubrication stage, as supported by the two-orders decrease in the interfacial true shear stress. This can be taken into account by considering that the PDMS-gel frictional dissipation, related to the bonding-debonding process (Schallamach process) of polymer chains onto the gel surface, must be expected to occur with a magnitude depending on the locally-averaged interfacial separation, which in turn depends on the local electrostatic interaction. Hence we find (Supplemental Section 4) a proportionality between the shear stress and the number of active bonds $N(\mathrm{pH})$, with

$$
N(p H)=N_{0} e^{-R_{b}^{3}\left(p_{r}-\sigma_{0}\right) / k_{B} T}
$$

where $R_{\mathrm{b}}$ is the polymer blob radius and $\sigma_{0}$ the externally applied pressure. The exponential takes approximately into account a random distribution of blob radius with root mean square radius $R_{\mathrm{b}}$ (from a standard random process well known in contact mechanics, see Supplementary Section 4). Figure 6d shows that $p_{\mathrm{r}}$ has a linear variation with $\mathrm{pH}$. By neglecting the contact area dependence of the friction (for hydrogel contact, there is a further contact area reduction related to the hydration and consequent reduction of the superficial polymer density), in Figure 6e we compare our theoretical prediction with the friction measurements in boundary regime at different $\mathrm{pH}$ values of the solution. The rather good comparison suggests that the hydration lubrication, originating in the double layer formation and from the consequent substantial drop of the number of active bonding sites [from $N_{0}$ into $N(\mathrm{pH})$ ], has a strong effect in term of friction reduction. In our case, a simple $\mathrm{pH}$ variation of the solution can be (relatively) easily adopted to activate (basic-pH) or deactivate (acid-pH) the double layer. 

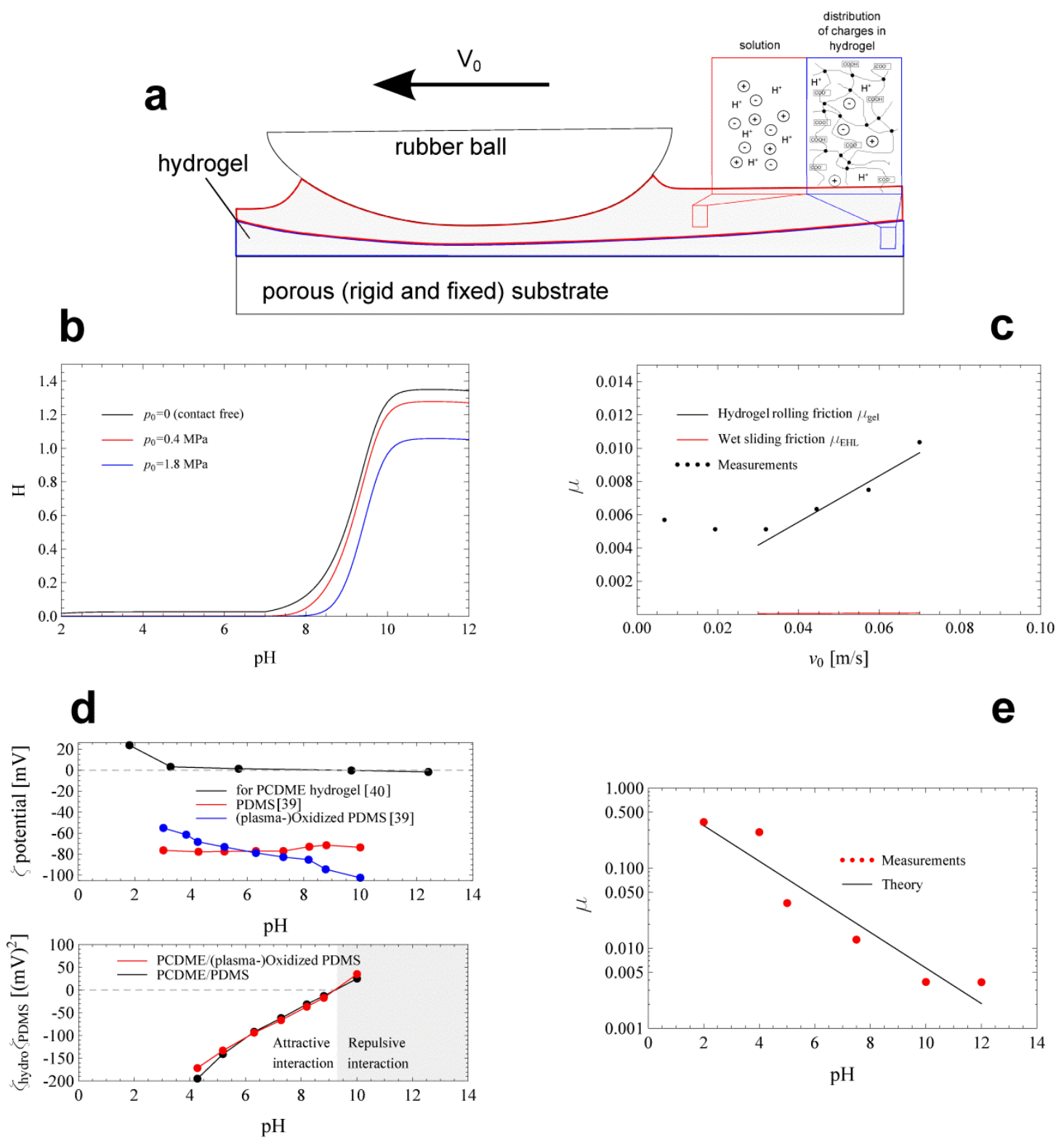

Figure 6: (a) Schematic of the contact model. (b) Hydration as a function of the $\mathrm{pH}$, for a $c_{\text {smo }}=1.8 \mathrm{M}, E_{\text {hydro }}=2.7 \mathrm{MPa}, p K_{d-\mathrm{COOH}}=5$ and a bath temperature of $T=330 \mathrm{~K}$. For squeezing pressure $p_{0}=0,0.4,1.8 M P a$. (c) Hydrogel rolling friction $\mu_{g e l}$ as a function of the sliding velocity $v_{0}$. For $\tau_{r} \approx 10^{-4} s$ and $R_{b}=3 \mathrm{~mm}$. (d) Top: Surface potential as a function of the solution $\mathrm{pH}$, for exposed PDMS (red curve, adapted from Ref. ${ }^{[42]}$ ), for plasma-oxidized PDMS (blue, adapted from Ref. ${ }^{[42]}$ ) and for a PCDME hydrogel (black, adapted from Ref. ${ }^{[41]}$ ); Bottom: Electrostatic interaction (proportional to $\zeta_{\text {hydro }} \cdot \zeta_{P D M S}$ ) as a function of the solution 
pH. (e) Experimental (dots) and theoretical predictions of the boundary friction as a function of $\mathrm{pH}$.

\section{Conclusion}

In conclusion, inspired by the natural lubrication mechanism of the eel, we created a new composite interface by a soft/hard combination strategy, obtained by $\mu \mathrm{m}$-sized (acrylic acid) hydrogel layer bonded onto a rigid, healing-initiator nonporous substrate. We show that such an interface allows for rapidly tunable, robust, self-healing and ultra-low friction $(\approx 0.01)$ characteristics, even under heavily loaded environment $(40 \mathrm{~N})$. Moreover, the $\mathrm{pH}$ responsiveness of the hydrogel, based on the swollen and contracted state by the acid-basic actuation, allows for a fast controllable friction switching between relatively high to ultra-low friction values. On the theoretical side, a novel scaling model of hydrogel lubrication has been derived which accurately captures the main (complex) physics occurring in such systems. Our findings definitely open a route for designing new friction-tunable intelligent devices for usage in mass transportation and chemical actuation. More importantly, the design concept originating from this novel composite interface has important implications and provides inspiration for the development of novel bio-mimetic cartilage articular materials.

\section{Experimental Section}

Experimental materials and methods are available in the supporting information.

\section{Supporting Information}

Supporting information is available via the internet at http://pubs.acs.org. 
Acknowledgements: This work was financially supported by NSFC (20434009, 21125316, 51335010) and Key Research Program of CAS (KJZD-EW-M01). DD acknowledges the support of the UK Engineering and Physical Sciences Research Council (EPSRC) via the Platform Grant EP/G026114/1.

\section{Reference}

[1] W. Barthlott, C. Neinhuis, Planta 1997, 202, 1.

[2] M. Nosonovsky, B. Bhushan, Curr. Opin. Colloid Interface Sci. 2009, 14, 270.

[3] T. L. Sun, L. Feng, X. F. Gao, L. Jiang, Acc. Chem. Res. 2005, 38, 644.

[4] S. Wooh, J. H. Koh, S. Lee, H. Yoon, K. Char, Adv. Funct. Mater. 2014, 24, 5550.

[5] B. N. J. Persson, Surf. Sci. Rep. 2006, 61, 201.

[6] Y. Tian, N. Pesika, H. B. Zeng, K. Rosenberg, B. X. Zhao, P. Mcguiggan, K. Autumn, J. Israelachvili, Proc. Natl. Acad. Sci. U. S. A. 2006, 103, 19320.

[7] D. M. Drotlef, P. Blumler, A. Del Campo, Adv. Mater. 2014, 26, 775.

[8] B. Bhushan, Philos. Trans. R. Soc., A 2009, 367, 1445.

[9] M. Spinner, A. Kovalev, S. N. Gorb, G. Westhoff, Sci. Rep. 2013, 3, 1846.

[10] C. Müller, A. Müller, T. Pompe, Soft Matter 2013, 9, 6207.

[11] D. Lü, C. Luo, C. Zhang, Z. Li, M. Long, Biomaterials 2014, 35, 3945.

[12] A. B. Faia-Torres, S. Guimond-Lischer, M. Rottmar, M. Charnley, T. Goren, K. ManiuraWeber, N. D. Spencer, R. L. Reis, M. Textor, N. M. Neves, Biomaterials 2014, 35, 9023.

[13] L. Major, J. M. Lackner, B. Major, $R S C A d v$. 2014, 4, 21108.

[14] T. A. Schmidt, N. S. Gastelum, Q. T. Nguyen, B. L. Schumacher, R. L. Sah, Arthritis Rheum. 2007, 56, 882.

[15] M. Chen, W. H. Briscoe, S. P. Armes, J. Klein, Science 2009, 323, 1698. 
[16] A. Li, E. M. Benetti, D. Tranchida, J. N. Clasohm, H. Schonherr, N. D. Spencer, Macromolecules 2011, 44, 5344.

[17] N. Maeda, N. Chen, M. Tirrell, J. N. Israelachvili, Science 2002, 297, 379.

[18] R. Tadmor, J. Janik, J. Klein, L. J. Fetters, Phys. Rev. Lett. 2003, 91, 115503.

[19] J. H. H. Bongaerts, J. J. Cooper-White, J. R. Stokes, Biomacromolecules 2009, 10, 1287.

[20] Y. Pan, Y. D. Xiong, Wear 2009, 266, 699.

[21] B. N. J. Persson, M. Scaraggi, J. Phys.: Condens. Matter 2009, 21, 185002.

[22] J. Klein, Science 2009, 323, 47.

[23] C. P. Neu, K. Komvopoulos, A. H. Reddi, Tissue Eng., Part B 2008, 14, 235.

[24] I. L. Kim, R. L. Mauck, J. A. Burdick, Biomaterials 2011, 32, 8771.

[25] Q. Wei, M. Cai, F. Zhou, W. Liu, Macromolecules 2013, 46, 9368.

[26] F. Li, Y. Su, J. Wang, G. Wu, C. Wang, J. Mater. Sci.: Mater. Med. 2010, 21, 147.

[27] R. Ma, D. Xiong, F. Miao, J. Zhang, Y. Peng, J. Biomed. Mater. Res., Part A 2010, 93, 1016.

[28] J. P. Gong, T. Kurokawa, T. Narita, G. Kagata, Y. Osada, G. Nishimura, M. Kinjo, J. Am. Chem. Soc. 2001, 123, 5582.

[29] J. P. Gong, Y. Katsuyama, T. Kurokawa, Y. Osada, Adv. Mater. 2003, 15, 1155.

[30] Q. Wang, J. L. Mynar, M. Yoshida, E. Lee, M. Lee, K. Okuro, K. Kinbara, T. Aida, Nature 2010, 463, 339.

[31] P. Lin, S. Ma, X. Wang, F. Zhou, Adv. Mater. 2015, 27, 2054.

[32] T. S. Wong, S. H. Kang, S. K. Y. Tang, E. J. Smythe, B. D. Hatton, A. Grinthal, J. Aizenberg, Nature 2011, 477, 443.

[33] B. Bhushan, J. N. Israelachvili, U. Landman, Nature 1995, 374, 607.

[34] Bo N. J. Persson, J. Chem. Phys. 2001, 115, 3840.

[35] E. Okamoto, H. Kasahara, A. Chiba, M. Taniguchi, E. Saitoh, Fish. Sci. 2009, 75, 463. 
[36] Y. Wu, X. W. Pei, X. L. Wang, Y. M. Liang, W. M. Liu, F. Zhou, NPG Asia Mater. 2014, 6, e136.

[37] Y. J. Lee, P. V. Braun, Adv. Mater. 2003, 15, 563.

[38] F. D. Jochum, P. Theato, Chem. Soc. Rev. 2013, 42, 7468.

[39] M. Ma, L. Guo, D. G. Anderson, R. Langer, Science 2013, 339, 186.

[40] M. Scaraggi, B. N. J. Persson, Tribol. Int. 2014, 72, 118-130.

[41] J. Ahmed, H. Guo, T. Yamamoto, T. Kurokawa, M. Takahata, T. Nakajima, J. P. Gong, Macromolecules 2014, 47, 3101-3107.

[42] P. Sun, J. H. Horton, Appl. Surf. Sci. 2013, 271, 344-351. 
The table of contents: A novel composite surface of ordered nanohydrogel arrays confined in anodic aluminum oxide template based on a soft/hard combination strategy is reported, which shows low friction coefficient $(<0.01)$ under $40 \mathrm{~N}$ and contact pressures $\sim 2 \mathrm{MPa}$, along with desirable regenerative and anti-wear properties, while exhibits smart switching between high friction $(>0.3)$ and super-lubrication $\left(\sim 10^{-3}\right)$ by acid-basic actuation.

Keyword: Nano-porous, Gel fibers array, Tunable friction/lubrication, Regenerable

Author: Shuanhong Ma, M. Scaraggi, Daoai Wang, Xiaolong Wang, Yongmin Liang,

Weimin Liu, D. Dini * and Feng Zhou*

Nano-porous substrate-infiltrated hydrogels: a bio-inspired regenerable surface for high load bearing and tunable friction

ToC figure

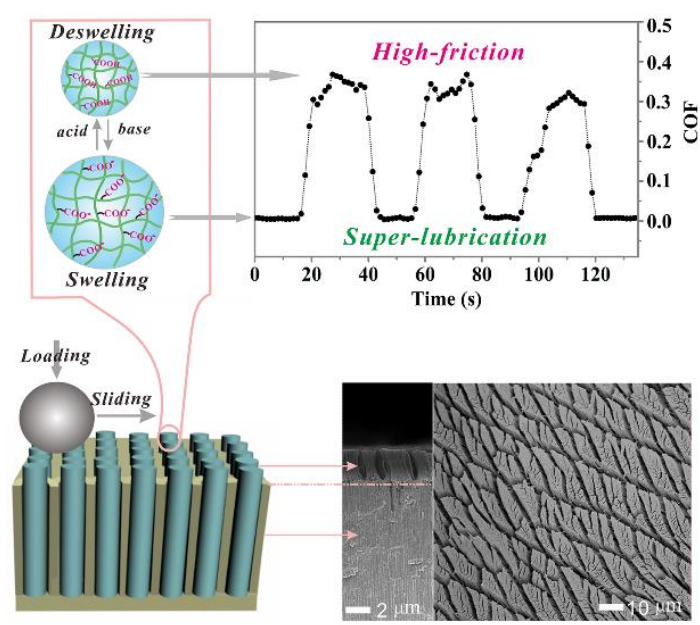

